\title{
Active Contour driven by geometric mean and standard deviation- based energy fitting model for the left ventricle segmentation from cardiac magnetic resonance images
}

\author{
K. Sivakumar ${ }^{1}$, Jayashree. $S^{2}$, Kaavya.K ${ }^{3}$, Pooja. $S^{4}$ \\ ${ }^{1}$ Asst. Prof., Department of Electronics and Communication Engineering \\ Rajalakshmi Institute of Technology, Chennai, India. \\ ${ }^{2,3,4}$ UG Students, Department of Electronics and Communication Engineering \\ Rajalakshmi Institute of Technology, Chennai, India.
}

sivakumar.k@ritchennai.edu.in, pooja.s.2017.ece@ritchennai.edu.in, kaavya.k.2017.ece@ritchennai.edu.in. jeyashree.s.2017.ece@ritchennai.edu.in

\begin{abstract}
This paper proposes a geometric mean and standard deviation-based energy fitting model to improve the accuracy of segmentation of the left ventricle from cardiac Magnetic Resonance Imaging (MRI). Energy-fitting-based active contour models emerged so far suffers either from intensity inhomogeneity or gives wrong segmentation result due to an inappropriate initial contour. Thus, an accurate and robust segmentation of the left ventricle from cardiac MRI still a challenging problem. Therefore, to tackle both the problems, a geometric mean-based energy-fitting model is proposed. Unlike the recent energy-fitting-based models which use the arithmetic mean to calculate the local energy, the proposed method uses geometric mean and scaled standard-deviation to compute the energy functional which drives the active contour to the region of interest. In addition to that completely removes the initial contour problem by automating it according to the input. The initial contour in the proposed model is a circle its radius and the centre are calculated from the input sample itself. This initial contour is an appropriate and automated that helps to reduce the computation time for segmentation. Experiments are conducted on cardiac MRI images the result obtained are compared with ground truth and evaluated by Average perpendicular distance (APD) and DICE similarity coefficient. Further the visual as well as evaluated parameters evidences that the proposed model performs better than the existing methods.
\end{abstract}

Keywords:Minimum Error Thresholding, Energy fitting, Centroid, Geometric Mean, Standard deviation, and Active Contour.

\section{INTRODUCTION}

Problems like coronary heart disease are treated well with the help of modern imaging modality like Medical Resonance Imaging (MRI), which is one of the medical imaging modalities widely used in medical imaging that helps the radiologist to find the problems in vivo heart and also to take right decision in shortest time [1]. The Segmented Left Ventricle $(\mathrm{LV})$ is used in assessing the cardiac functionality which is essential in today's highly populated world for the timely diagnosis of the disease and for treatment planning [2].Hence, in recent years many researchers have proposed a variety of energy-fitting-based Active Contour Model (ACM) for the segmentation of LV.

ACMs are broadly classified into two categories that are edge based and region based. The edge-based ACMs [3-9], drives the curve to the Region of Interest (ROI) based the edge information while the region-based ACMs [10-27], use either local and/or global information, to drive the curve to the ROI. For instance, the C-V model [10], a popular region-based model for segmentation that segments the ROI well but fails when the image is having intensity inhomogeneity, the reason behind is that the model assumes that the regions within and out the contour $C$ are homogeneous. To overcome this limitation Piecewisesmooth model [12] was introduced, it equally performs as [10] but the computational efficiency is less. Region Scalable Fitting (RSF) energy model [13] was introduced to manage the limitation of [10], it uses the local image information obtained via two predefined functions by which handles the problem of intensity inhomogeneity but updating of the predefined functions at every iteration leads to high computational cost. ACM driven by Local Image Fitting (ACM-LIF) model [15]considers the limitation of [13] and reduces the computational cost to half but the segmentation result depends on the position of the initial contour. 
As [15] a local region-based CV model [16] was proposed with less computational cost than that of [13] but its performance depends on the initial contour. The ACM driven by Local Histogram Fitting (ACM-LHF) [18] shows its performance in handling intensity inhomogeneity problem. The computation of histogram for both background and ROI is the reason behind for its high computational cost.

Recently for an accurate and robust LV segmentation, ACM driven by Local PreFitting (ACM-LPF) energy model [25] was proposed which uses an idea of computing local information before curve evolution thereby avoids the time-consuming operation that is updating of the function at every iteration. This idea reduces the computation time of the model, but it is still sensitive to initial values. The ACM guided by Global and Local Signed Energy based Pressure Force (ACM-GLSE-PF) model [26] tries to overcome the limitations of [10] as well as [13] but the weights used to balance the propagation function makes the model to fall under the category of high computational cost. In [27] a combination of standard deviation and arithmetic mean is used to develop the energy functional this new combination handles the problem of intensity inhomogeneity but updating of weight at every iteration is the limitation of the model.

Overall, in general, all the ACM-driven energy-fitting-based models [10-27], are fails to balance the following (i) handling intensity inhomogeneity, (ii) initial contour, (iii) computation time and (iv) an appropriate assumption of initial values. Therefore, to develop a model which can solve these two problems simultaneously and segment the LV accurately is necessary. To provide a solution to the above-mentioned problems a Geometric Mean Based-Energy-Fitting (GMEF) model for accurate, robust, and fast segmentation of the LV from cardiac MRI images is proposed in this paper. In general, when compared with arithmetic mean the geometric mean is preferred reason is that the geometric mean-based processing loses less data than the arithmetic mean-based processing. Hence the proposed model uses the geometric mean along with adaptively scaled standard deviation to develop the energy functional. Further, image information used to develop the energy function needs no update, it is computed only once before the curve evolution. In addition to that to overcome the problem of initial contour, an appropriate and automated initial contour is introduced. Hence the proposed GMEF is completely insensitive to the initial contour problem. The proposed model first automates the initial contour, described in section 3.1, then with the initial contour and the initialvalues the proposed energy function drives the contour to segments the LV from cardiac MRI, described in 3.2.

The complete work of this paper is divided into five sections. Section 2 briefs related with and its limitations, section 3 elaborates the GMEF model, section 4 - details the algorithm, experimental result and discussion, and section 5 Concludes the work.

\section{RELATED WORK}

\section{A. Region Scalable Fitting (RSF) energy model}

Chunming Li et al [13] proposed the following RSF energy model,

$$
\begin{aligned}
& \left.E^{R S F}\left(\phi, f_{1}(x), f_{2}(x)\right)=\sum_{i=1}^{2} \lambda_{i} \int_{\Omega} K_{\sigma}(x-y)\left|I(y)-f_{i}(x)\right|^{2} M_{i}(\phi(y)) d y\right) d x \\
& \int_{\Omega} \delta_{\varepsilon}(\phi(x))|\nabla \phi(x)| d x \\
& \int_{\Omega} \frac{1}{2}(|\nabla \phi(x)|-1)^{2} d x
\end{aligned}
$$

Functions (2) and (3) respectively serves the purpose of smoothing and regularizing the evolving curve. Hence with smoothing and regularizing term the RSF energy functional can be written as,

$$
\begin{gathered}
\left.E^{R S F}\left(\phi, f_{1}(x), f_{2}(x)\right)=\sum_{i=1}^{2} \lambda_{i} \int_{\Omega} K_{\sigma}(x-y)\left|I(y)-f_{i}(x)\right|^{2} M_{i}(\phi(y)) d y\right) d x+ \\
v \int_{\Omega} \delta_{\varepsilon}(\phi(x))|\nabla \phi(x)| d x+\mu \int_{\Omega} \frac{1}{2}(|\nabla \phi(x)|-1)^{2} d x
\end{gathered}
$$

Where, $x, y \in \Omega, \lambda_{i}, v$ and $\mu$ are positive constant. $K_{\sigma}$ - Kernel function $M_{1}(\phi)=H(\phi)$ and $M_{2}(\phi)=1-H(\phi)$. 
$H$ is the Heaviside function and is approximated by smooth function $H_{\epsilon}$.

$H_{\epsilon}=\frac{1}{2}\left[1+\frac{2}{\pi} \arctan \left(\frac{x}{\epsilon}\right)\right]$

The derivate of $H_{\epsilon}$, is the Dirac function $\delta_{\epsilon}(x)$ which is defined by,

$\delta_{\epsilon}(x)=\frac{\epsilon}{\pi\left(\epsilon^{2}+x^{2}\right)}$

keeping $\phi$ as constant, functions $f_{1}(x)$ and $f_{2}(x)$ are obtained by minimizing (4) that are given by.

$$
\begin{aligned}
& f_{1}(x)=\frac{\int_{\Omega} K_{\sigma}(x-y)\left[H_{\epsilon}(\phi(y)) I(y)\right] d y}{\int_{\Omega} K_{\sigma}(x-y) H_{\epsilon}(\phi(y)) d y} \\
& f_{2}(x)=\frac{\int_{\Omega} K_{\sigma}(x-y)\left[1-H_{\epsilon}(\phi(y)) I(y)\right] d y}{\int_{\Omega} K_{\sigma}(x-y)\left[1-H_{\epsilon}(\phi(y))\right] d y}
\end{aligned}
$$

Keep the functions $f_{1}(x)$ and $f_{2}(x)$ as constant then minimise (4), with respect to $\phi$, the equation obtained is

$$
\frac{\partial \phi}{\partial t}=-\delta_{\epsilon}(\phi)\left(\lambda_{1} e_{1}-\lambda_{2} e_{2}\right)+v \delta_{\epsilon}(\phi) \operatorname{div}\left(\frac{\nabla \phi}{|\nabla \phi|}\right)+\mu\left(\nabla^{2} \phi-\operatorname{div}\left(\frac{\nabla \phi}{|\nabla \phi|}\right)\right)
$$

Where, $e_{1}$ and $e_{2}$

$$
e_{i}(x)=\int K_{\sigma}(y-x)\left|I(x)-f_{i}(y)\right|^{2} d y
$$

\section{B. ACM driven by Local Pre-Fitting (LPF) Energy model}

Keyan Ding et al [25] proposed LPF model. The energy functional defined by [25] is $E^{L P F}\left(\phi, f_{s}(x), f_{l}(x)\right)=\int_{\Omega}\left(\int_{\Omega} K_{\sigma}(x-y)\left|I(y)-f_{s}(x)\right|^{2} H_{\varepsilon}(\phi(y)) d y\right) d x+$

$\int_{\Omega}\left(\int_{\Omega} K_{\sigma}(x-y)\left|I(y)-f_{l}(x)\right|^{2}\left(1-H_{\varepsilon}(\phi(y))\right) d y\right) d x$

Where, $K_{\sigma}$ is the gaussian kernel, and $\sigma$ is the standard deviation used to control the size of the window. Functions (2) and (3) are added with (11) to maintain smoothness as well as to regularize the evolving curve. Size of the window is

$W_{l}=4 * \sigma+1$

Where $W_{l}$ - the window length. Let the $\Omega_{x}$ represents a small neighborhood centred at $x$ with radius $w, \Omega s$ and $\Omega_{l}$ represents the regions its average intensity lower than and higher than the average intensity in the region $\Omega_{x}$ respectively. These regions,,$\Omega s$ and $\Omega_{l}$, are defined as follows

$\Omega_{s}=\left\{y \mid I(y)<f_{m}(x)\right\} \cap \Omega_{x}$

$\Omega_{l}=\left\{y \mid I(y)>f_{m}(x)\right\} \cap \Omega_{x}$

The functions for the regions $\Omega_{x}, \Omega s$, and $\Omega_{l}$ respectively are defined by

$f_{m}(x)=\operatorname{mean}\left(I(y) \mid y \epsilon \Omega_{x}\right)$

$f_{s}(x)=\operatorname{mean}\left(I(y) \mid y \in \Omega_{s}\right)$

$f_{l}(x)=\operatorname{mean}\left(I(y) \mid y \in \Omega_{l}\right)$

With(15),(16) and (17) solving for evolving cure the following expression was obtained using gradient descent flow,

$$
\frac{\partial \phi}{\partial t}=-\delta_{\epsilon}(\phi)\left(e_{s}(x)-e_{l}(x)\right)+v \delta_{\epsilon}(\phi) \operatorname{div}\left(\frac{\nabla \phi}{|\nabla \phi|}\right)+\mu\left(\nabla^{2} \phi-\operatorname{div}\left(\frac{\nabla \phi}{|\nabla \phi|}\right)\right)
$$

Where, $e_{s}(x)$ and $e_{l}(x)$ are given by

$$
\begin{aligned}
& e_{s}(x)=\int K_{\sigma}(y-x)\left|I(x)-f_{s}(y)\right|^{2} d y \\
& e_{l}(x)=\int K_{\sigma}(y-x)\left|I(x)-f_{l}(y)\right|^{2} d y
\end{aligned}
$$

The major difference between [13] and [25] is in the method of computation of local information to construct the energy fitting functional. The functions (7) and (8) in [13] are used to smooth the intensities in and outside of the contour C. It is apparent that updating of (7) and (8) at every iteration is required which result in high computational cost. To overcome the limitation of updating the function at every iteration [25] introduced a set of functions (15), (16) and (17). These functions are computed before the curve evolution hence updating of these functions at every iteration is not necessary which makes the method better than [13] but it is sensitive to initial values. Hence, the GMEF model is proposed which has the capability of managing (i) the computation time, (ii) sensitiveness to initial value, initial contour, and (iii) the intensity inhomogeneity. Section 3 describes the proposed model. 


\section{THE PROPOSED METHOD}

This section describes a method of finding the initial values, initial contour, and scaling factor from a blob of the binarized input image. The properties like perimeter, centroid, and eccentricity of the blob are obtained and used to find an appropriate initial value that are having a significant impact on the output, the initial contour, and the scaling parameter, $\alpha$.

\section{A. The method for initial contour automation}

As mentioned in the introduction part the accuracy of segmentation in most of the existing ACMs also depends on the initial contour. To overcome this limitation the idea used in this paper is that the input image is binarized first using Minimum Error Thresholding (MET), comparing with other thresholding methods MET works well for the application of LV segmentation. The obtained binarized image is having many blobs due to pixels having intensities nearly equal to pixel intensity of LV, white patches, as shown in figure 1 (b). Hence to choose the blob which indicates the LV and to neglect the remaining blobs the Max function is used with the area as its arguments. Let $A_{1}, A_{2} \ldots \ldots . A_{n}$ be the area of the blobs in the binary image, figure 1 (b), from which a bigger blob, $B_{b}$, is identified by using the max function.

$$
B_{b}=\max \left(A_{1}, A_{2} \ldots \ldots . A_{n}\right)
$$

The max function gives the bigger blob, $B_{b}$, as shown in figure 1(c) from the binarized image. Area and perimeter are calculated from $B_{b}$ then used in (28) and (30) to calculate the value of $\lambda_{1}$ and $\alpha$ respectively. In fact, the aim of this section is to describe the method of finding an appropriate initial contour. A small rectangle or square, in general, can be used as an initial contour but in this work small circle with $r$ is used as initial contour. Using the perimeter, $p$, of $B_{b}$ the initial contour's radius is calculated by

$$
r=0.5 * \frac{p}{2 * \pi}
$$

Centroid of $B_{b}$ acts as a centre for initial contour. Hence with radius $r$ from (22) and the centroid automatically constructed initial contour is shown in figure 1(d).
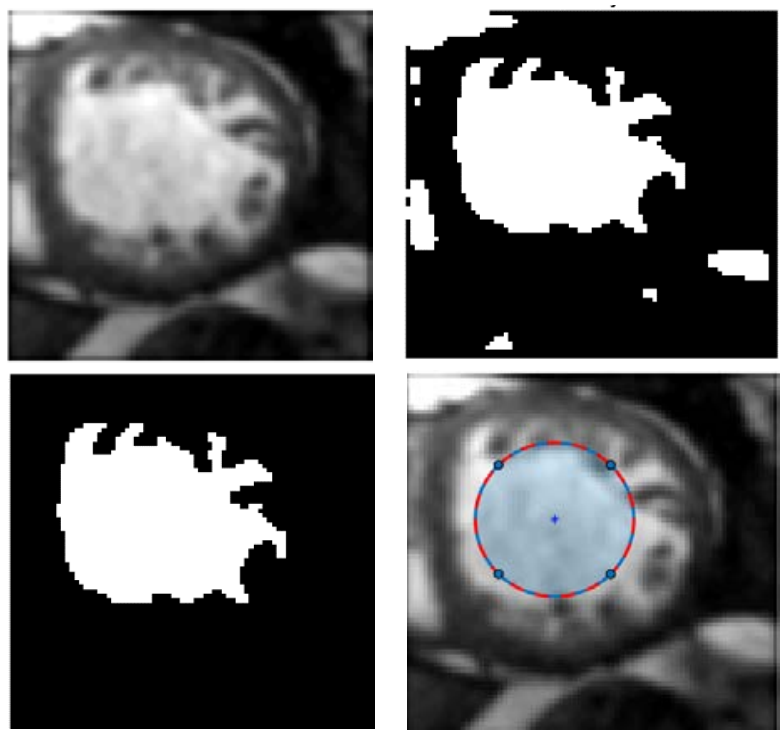

Figure 1 (a) Top Left-Input Image, (b) Top Right-Binarized image by MET, (c) Bottom left- Biggest Blob, and (d) Bottom right- Input with automatically constructed initial contour (red in colour)

\section{B. The proposed energy functional}

As used in ACMs, the proposed method also uses the initial values to evolve the curve. Existing ACMs use the fixed initial values to evolve the curve. However, there are some initial values that have an effect on the segmentation result if such values are adjusted according to the input the chances of improving the accuracy of the segmentation result are more. Therefore, in the proposed model such initial values are autotuned according to the input by making use of the properties of $B_{b}$ thereby tries to improve the segmentation result. The proposed function used to increase the segmentation accuracy is described as follows 
Let $\Omega \subset R^{2}$ be the image domain and $I: \Omega \subset R$ be the input (gray) image. The proposed method defines the following functions to overcome the limitations of [13] and [25]

$f_{g m}(x)=\operatorname{geomean}\left(I(y) \mid y \epsilon \Omega_{x}\right)+\alpha \cdot \operatorname{std}\left(I(y) \mid y \epsilon \Omega_{x}\right)(23)$

$f_{s}(x)=\operatorname{mean}\left(\left(I(y) \mid y \in \Omega_{s}\right)\right.$

$f_{l}(x)=\operatorname{mean}\left(I(y) \mid y \in \Omega_{l}\right.$

Where std(I(y)) - standard deviation of intensity within the region $\Omega_{x}$. The significance of the standard deviation function is explained in the experimental result and discussion. $\alpha-$ is a small fraction that is calculated using (30) and used to scale the standard deviation. Further, $\Omega_{x}$ - a local region with $x$ at its centre, $\Omega_{s}$ and $\Omega_{l}$ are respectively the region with average intensity lesser and greater than the average intensity in $\Omega_{x}$ respectively.

The proposed method uses (23), (24) and (25), then defines the following energy functional. As it is done in all ACMs, to keep the curve smooth and to avoid reinitialization at every iteration the curve smoothing, and the regularization term are added. Complete energy functional of the proposed model is given by

$$
\begin{aligned}
& E^{G M E F-A C M}\left(\phi, f_{s}(x), f_{l}(x)\right)= \\
& \lambda_{1} \int_{\Omega}\left(\int_{\Omega} K_{\sigma}(x-y)\left|I(y)-f_{s}(x)\right| H_{\varepsilon}(\phi(y)) d y\right) d x+\lambda_{2} \int_{\Omega}\left(\int_{\Omega} K_{\sigma}(x-y)\left|I(y)-f_{l}(x)\right|(1-\right. \\
& \left.\left.H_{\varepsilon}(\phi(y))\right) d y\right) d x+v \int_{\Omega} \delta_{\varepsilon}(\phi(x))|\nabla \phi(x)| d x+\mu \int_{\Omega} \frac{1}{2}(|\nabla \phi(x)|-1)^{2} d x
\end{aligned}
$$

Minimizing (24) using gradient descent flow the following expression is obtained,

$\frac{\partial \phi}{\partial t}=-\delta_{\epsilon}(\phi)\left(\lambda_{1} e_{s}(x)-\lambda_{2} e_{l}(x)\right)+v \delta_{\epsilon}(\phi) \operatorname{div}\left(\frac{\nabla \phi}{|\nabla \phi|}\right)+\mu\left(\nabla^{2} \phi-\operatorname{div}\left(\frac{\nabla \phi}{|\nabla \phi|}\right)\right)$

Where, $v$ and $\mu$ are positive constant. And $e_{s}$ and $e_{l}$ in (24) are already defined in (19) and (20). $\lambda_{1}$ and $\lambda_{2}$ are the weights for first two teams in the energy function which are getting calculated automatically from the input image, while most of ACMs assumes $\lambda_{1}=\lambda_{2}=1$. This auto tuned $\lambda^{\prime} s$ play a significant role in accurate segmentation of LV. The value of $\lambda^{\prime} s$ are calculated as follows

$\lambda_{1}=5 * \frac{A_{b}}{p}$

$\lambda_{2}=\frac{1}{2} \lambda_{1}$

Where, $A_{b}, p$ are the area, and the perimeter of the $B_{b}$ respectively. The function (26) is the major contribution of this paper. The reason behind the use of geometric mean instead of arithmetic mean, most of the ACMs uses arithmetic mean, is that geometric mean retains more (image) details even in the presence of noise. Further geometric mean helps to neglect the outliers that is the effect of noise in the noisy image, whereas arithmetic mean simply finds the mean of every pixels in the image. Replacement of arithmetic mean in (15) by geometric mean and adding a scaled standard deviation shows a considerable improvement in the accurate segmentation of LV.

\section{Computation of Scaling parameter.}

To improve the LV segmentation accuracy a standard deviation of the pixels within the region $\Omega_{x}$ is calculated which is scaled with $\alpha$ then added with geometric mean of the pixels within the region $\Omega_{x}$. The scaling parameter $\alpha$ controls the amount of standard deviation which is being added with geometric mean. Using $A_{b}, p$ of $B_{b}$ the scaling factor $\alpha$ is calculated by

$\alpha=\frac{A_{b}}{10 * p}$

\section{IMPLEMENTATION, EXPERIMENTAL RESULT, AND DISCUSSION}

\section{A. Algorithm of the proposed model}

Section 3.1 gives the method of computing the initial contour, which is the major problem in many of the ACM-energy fitting models, and initial values which help to improve the segmentation result are described in section 3.2 and 3.3. And this section describes the implementation steps of the proposed model.

Proposed algorithm: ACM-GMEF

Aim: To segment accurately and robustly the LV from cardiac MRI Image 
Input: Cardiac MRI Image and Segmented ground truth

Output: Segmented Image.

Step 1: Get the input image then binarize it by MET.

Step 2: Find the $B_{b}$ using equation (21)

Step 3: Measure the properties of $B_{b}$

Step 4: With the centroid of $B_{b}$ as the center, create the initial contour with the radius calculated using the equation (3)

Step 5: Set up the initial values $v, \mu, \sigma, \varepsilon$ and time step $\Delta t$ which are not required to tune.

Step 6: Compute window length $W_{l}$ using (11), $\lambda_{1}$ and $\lambda_{2}$ using (28) \& (29) and $\alpha$ using (30)

Step 7: Define $f_{g m}(x), f_{s}(x) \& f_{l}(x)$

Step 8: Use the initial values given in step 5 and step 6, compute functions $e_{s}(x)$ and $e_{l}(x)$ using (19) \& (20).

Step 9: For $i=1: N \quad / /$ Where $N$ - number of iterations.

update the level set function (27) then find the ROI.

End

Step 10: Compare the Obtained ROI from step 9 with ground truth.

Step 11: Calculate the evaluation parameters, DICE and APD.

Experiments are conducted to test the performance of the proposed model for various inputs are compared with recent ACMs. The figure 2 shows the segmentation result for RSF, LIF, ACMLoG, ACM-LPF, ACLEPF, ACM-ALF and proposed model. The blue contour is the ground truth, and the red is the developed contour by each of the models. The images in the first row are the inputs used to test the segmentation performance. Second row to sixth row are the performances of each of the methods listed, the last row is the the performance of the proposed model. It can be seen from the figure that the proposed method gives an accurate result than RSF, LIF, ACM-LoG, ACM-LPF, ACLEPF, ACM-ALF in terms of visual.

\section{B. Experimental result}

The performance of each models is evaluated by two metrics which are the Average Perpendicular Distance (APD), and DICE coefficient. The parameters APD and the DICE are defined by

$\mathrm{APD}=\sqrt{\left(\text { etimated }_{x}-\text { groundtruth }_{x}\right)^{2}+\left(\text { estimated }_{y}-\text { groundtruth }_{y}\right)^{2}}$

Where, estimated $d_{x}$ is $x$-coordinate of estimated value, groudtruth $h_{x}$ is $x$-coordinate of ground truth and DICE coefficients defined by

DICE $=\frac{2\left(A_{1} \cap A_{2}\right)}{A_{1}+A_{2}}$

Where, $A_{1}$ and $A_{2}$ are the regions by ground truth and region by the model, respectively.

As it was already mentioned that the tuning of the initial parameters for the proposed model are an automated one, whereas in many of the existing ACMs tuning of initial parameters approximate its segmentation result with the ground truth. For instance, when the window scaling parameter $\sigma$ in LIF is increased to 20 its segmentation resultapproximates the proposed method. As the window size in LIF is $4 \sigma+1$ if $\sigma=20$ then the window size becomes $91 \times 91$ which is more or less equal to the size of the input image.

Hence, if $\sigma$ is increased to 20 then the LIF is no longer a local image fitting model rather it becomes a global image fitting energy model. However, the $\sigma$ value needs to be changed based on the nature of the input to approximate its result to the ground truth. The same problem exists for LBF, ACM-LoG models also and these models are also need tuning of $\sigma$ for better results. Since the initial values that need tuning according to the input are automated which helps the proposed model in another way to improve its performance in terms of segmentation accuracy. Other initial values which have not much impact on segmentation result are kept as constant as it was used in ACMs. The other initial values used in the proposed model are $\mu=1, v=0.02 \times 255^{2}$ time step size $\Delta t=0.1, \varepsilon=1$ and $\sigma=9$.To compare the proposed model's performance with RSF, LIF, ACM-LoG, ACM-LPF, ACLEPF, ACM-ALF by means of evaluation metrics five images were taken, shown in the first row of figure 2, which are base slice, apex slice, slice with highly intensity inhomogeneity, and slice with weak edges. In fact, all the images are having very weak edges. Evaluation parameters are tabulated for each of the input. It is Inferred from the table that 
Journal of University of Shanghai for Science and Technology ISSN: 1007-6735 higher the accuracy of segmentation when the APD is as low as possible and also DICE coefficient is near to one.

It is clear from table 1 that the proposed method's APD is minimum, and the DICE coefficient is maximum when compared with all other models for all the five input images.

Further to verify the consistency, speed of computation, and efficiency of the proposed model, one hundred images are taken as input which include apex, middle and base slices then the average APD and average DICE coefficient and average time are tabulated. The same experiment is conducted for the other models also. From the tabulated metrics, the table 2, it is confirmed that the proposed model performs better than all other models for almost all kind of image slices which is the strength of the proposed energy fitting model.

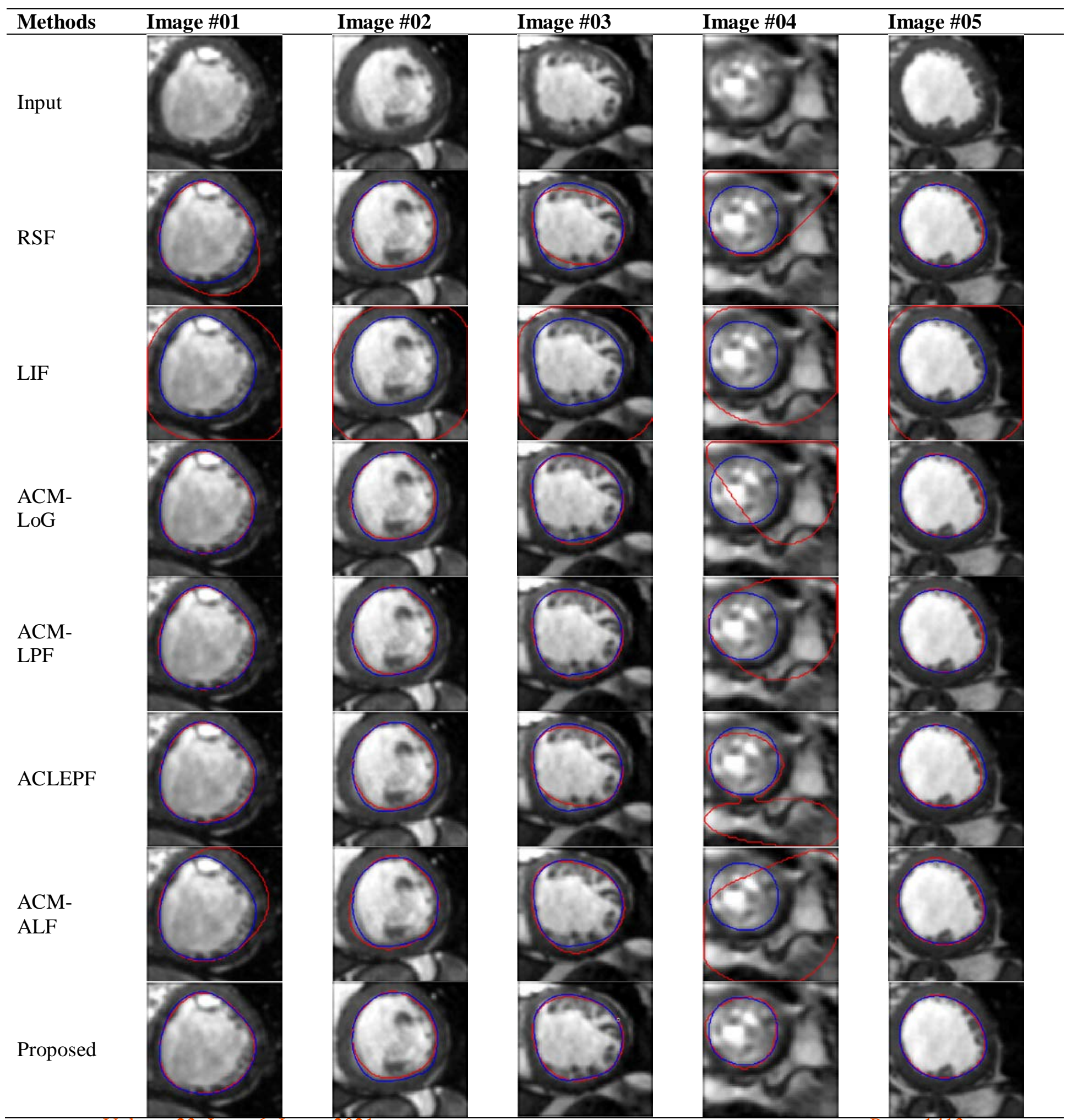


Figure 2. LV segmentation results of the RSF, LIF, ACM-LoG, ACM-LPF, ACLEPF, ACMALF and proposed model on five different of cardiac MRI Images. First row- Original Image (Input Image) and Second, Third, Fourth, Fifth, Sixth and Seventh row are the results of RSF, LIF, ACM-LoG, ACM-LPF, ACLEPF, ACM-ALF, respectively.

But it is not true for many of the existing models that when the image slice is a base slice even recently proposed models fails to segment it properly. Further to ensure the consistency of each of the methods the standard deviation is also tabulated along with average APD, DICE, and computation time. As the standard deviation is comparatively less for the proposed model than that of the recent models hence it is proved that the proposed model is consistent.

Table 1 Segmentation performance - Comparison of AC model for the selected input images

\begin{tabular}{|c|c|c|c|c|}
\hline Input Image & Methods & APD & DICE & $\begin{array}{c}\text { Computation time } \\
\text { (in seconds) }\end{array}$ \\
\hline \multirow{7}{*}{ Image \#01 } & RSF & 3.0166 & 0.9067 & 9.4712 \\
\hline & LIF & 13.4993 & 0.6310 & 0.2043 \\
\hline & ACM-LoG & 0.6005 & 0.9838 & 0.3214 \\
\hline & ACM-LPF & 0.6584 & 0.9812 & 0.1243 \\
\hline & ACLEPF & 0.7210 & 0.9807 & 4.9729 \\
\hline & ACM-ALF & 3.5375 & 0.8791 & 24.7102 \\
\hline & Proposed & 0.5296 & 0.9863 & 0.1141 \\
\hline \multirow{7}{*}{ Image \#02 } & RSF & 1.2885 & 0.9506 & 7.9660 \\
\hline & LIF & 17.0453 & 0.5297 & 0.2012 \\
\hline & ACM-LoG & 1.0341 & 0.9577 & 0.2068 \\
\hline & ACM-LPF & 1.0474 & 0.9588 & 0.0539 \\
\hline & ACLEPF & 1.1307 & 0.9555 & 4.7283 \\
\hline & ACM-ALF & 1.1500 & 0.9453 & 23.8820 \\
\hline & Proposed & 1.1362 & 0.9570 & 0.0339 \\
\hline \multirow{7}{*}{ Image \#03 } & RSF & 1.6914 & 0.9375 & 7.6023 \\
\hline & LIF & 17.1908 & 0.5281 & 0.1944 \\
\hline & ACM-LoG & 0.8877 & 0.9647 & 0.1949 \\
\hline & ACM-LPF & 0.8606 & 0.9637 & 0.0307 \\
\hline & ACLEPF & 1.3173 & 0.9527 & 3.8926 \\
\hline & ACM-ALF & 1.3668 & 0.9418 & 23.6397 \\
\hline & Proposed & 0.7335 & 0.9741 & 0.0327 \\
\hline \multirow{7}{*}{ Image \#04 } & RSF & 9.6820 & 0.6060 & 5.3331 \\
\hline & LIF & 15.1491 & 0.4139 & 0.1381 \\
\hline & ACM-LoG & 14.4164 & 0.3996 & 0.1738 \\
\hline & ACM-LPF & 13.3385 & 0.5079 & 0.0183 \\
\hline & ACLEPF & 12.7870 & 0.5309 & 3.3765 \\
\hline & ACM-ALF & 18.6494 & 0.3343 & 15.3255 \\
\hline & Proposed & 0.7562 & 0.9492 & 0.0282 \\
\hline \multirow{7}{*}{ Image \#05 } & RSF & 0.7170 & 0.9697 & 7.4232 \\
\hline & LIF & 19.0712 & 0.4850 & 0.1841 \\
\hline & ACM-LoG & 0.7321 & 0.9690 & 0.2257 \\
\hline & ACM-LPF & 0.6734 & 0.9691 & 0.0315 \\
\hline & ACLEPF & 1.0869 & 0.9563 & 3.8158 \\
\hline & ACM-ALF & 0.7656 & 0.9562 & 24.8290 \\
\hline & Proposed & 0.4833 & 0.9798 & 0.0372 \\
\hline
\end{tabular}

Table 2 - Comparison of Active contour models in terms of Mean and Standard deviation of APD, DICE and Computation time

\begin{tabular}{ccccccc}
\hline \multirow{2}{*}{$\begin{array}{c}\text { Name of the } \\
\text { Methods }\end{array}$} & \multicolumn{2}{c}{$\begin{array}{c}\text { Average Perpendicular } \\
\text { Distance } \\
\text { (APD) }\end{array}$} & \multicolumn{2}{c}{ DICE Similarity Coefficient } & \multicolumn{2}{c}{$\begin{array}{c}\text { Execution time } \\
\text { (in second) }\end{array}$} \\
\cline { 2 - 7 } & Mean & $\begin{array}{c}\text { Standard } \\
\text { deviation }\end{array}$ & Mean & $\begin{array}{c}\text { Standard } \\
\text { deviation }\end{array}$ & Mean & $\begin{array}{c}\text { Standard } \\
\text { deviation }\end{array}$ \\
\hline RSF & 3.82 & 4.9419 & 0.8439 & 0.2019 & 8.2371 & 1.9435 \\
LIF & 15.2436 & 3.9568 & 0.5355 & 0.1092 & 0.1855 & 0.0435 \\
ACM-LoG & 1.8599 & 3.1774 & 0.9073 & 0.1637 & 0.1892 & 0.0246 \\
ACM-LPF & 1.8532 & 3.0596 & 0.9114 & 0.1547 & 0.0283 & 0.0104 \\
Volume 23, Issue 6, June - 2021 & & & & & Page -1414
\end{tabular}



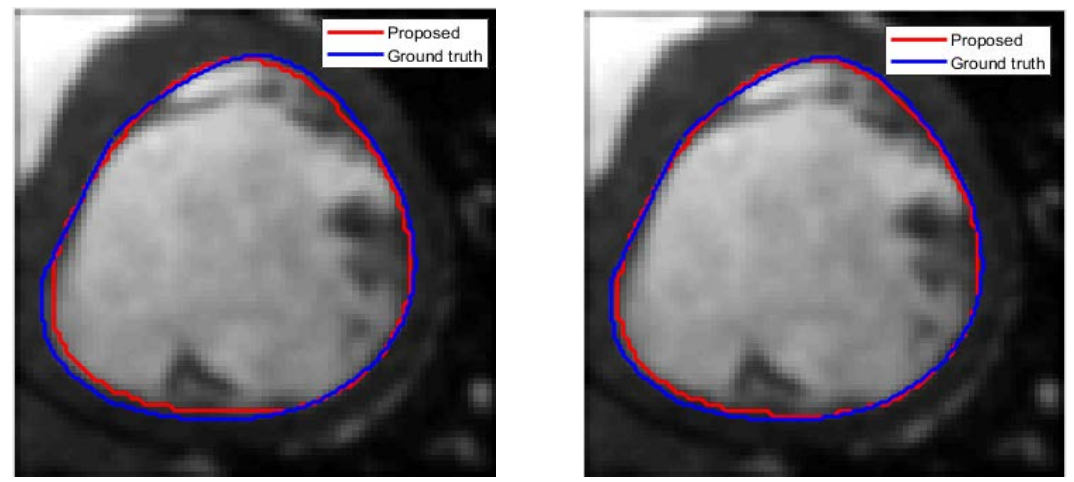

Figure 3 shows the result for $3\left(\right.$ a) fixed values of $\lambda^{\prime} s$, 3(b) tuned values of $\lambda^{\prime} s$

\section{Discussion on parameters and its impact on segmentation result.}

It is essential to know the reason for preferring the self- tuned $\lambda^{\prime} s$ rather than having fixed values to it. The existing ACMs use fixed $\lambda$ values. As stated already, figure (a) shows the segmentation result for $\lambda_{1}=\lambda_{2}=1$, that is for fixed values, based on it the obtained APD and DICE are 0.8903 and 0.9755 , respectively. Figure (b) shows the segmentation result for the $\lambda^{\prime} s$, which are calculated using (28) and (29), based on that respectively obtained APD and DICE are 0.6370 and 0.9832. Now it is clear from the APD and DICE values that the accuracy of segmentation increases due to automated values of $\lambda$ are used.

The scaling factor $\alpha$, equation (30), which scales the standard deviation is obtained locally and it is calculated automatically from the input and is having an impact on the segmentation result that is it reduces the computation time and increases the DICE metric. The objectives of having $\lambda_{1}$ and $\lambda_{2}$ and $\alpha$, are to improve the overall performance of the proposed model.

MATLAB 2018b was used to simulate both the proposed and the existing models with the systems specification of 12 GB ram, Intel Core i5, CPU @1.7Hz, 64-bit operating system.

The inputs are taken from https://www.cardiacatlas.org/studies/sunnybrook-cardiac-data/and http://www.cse.yo-rku.ca/ mrida-taset// used to validated the ACMs as well as the proposed model.

\section{CONCLUSION}

In this paper, a geometric mean-based energy fitting model has proposed to segment accurately the LV from cardiac magnetic resonance images. The scaling factor controls the amount of standard deviation to be added with the geometric mean. The addition of standard deviation with geometric mean helps the proposed model to handle intensity inhomogeneity. Since the initial contour is a small circle its centre and the radius are obtained automatically from the properties of the $B_{b}$ hence, the proposed model completely eliminates the initial contour problem and from the properties of $B_{b}$ self-tuned initial values are obtained which are aiding to improve the segmentation accuracy. Based on the evaluation parameter it is further proved that the proposed model gives better segmentation results than the existing ACMs. For the proposed model, the APD and DICE coefficient obtained respectively for an individual input are 0.4833 and 0.9798 . These values clearly tell that the proposed model segments well, while for the hundred inputs images the average APD and the average DICE coefficient respectively are 1.1049 and 0.9494 . This indicates that for few inputs the APD goes more than 1 and DICE goes less than 0.9. Hence, future work may be to bring the average APD less than 1 and maintain the DICE coefficient more than 0.97 by having energy function with suitable initial values.

\section{REFERENCES}

[1] https://www.nhlbi.nih.gov/health-topics/cardiac-mri 
[2] Dangi, S; Yaniv, Z; Linte, CA. Left ventricle segmentation and quantification from cardiac cine MR images via multi-task learning. International Workshop on Statistical Atlases and Computational Models of the Heart. 2018,16, 21-31.

[3] Caselles, V; Catté, F; Coll, T; Dibos, F. A geometric model for active contours in image processing. Numerischemathematik. 1993, 66(1),1-31

[4] Caselles, V; Kimmel, R; Sapiro, G. Geodesic active contours. International journal of computer vision. 1997, 22(1), 61-79.

[5] Y. Zheng, G. Li, X. Sun, X. Zhou, Fast edge integration based active contours for color images, Comput. Electr. Eng. 35 (2009) 141-149

[6] N. Paragios, R. Deriche, Geodesic active regions and level set methods for supervised texture segmetation, Int. J. Comput. Vision 46 (2002) 223-247

[7] R. Kimmel, A.M. Bruckstein, Regularized Laplacian zero crossings as optimal edge integrators, Int. J. Comput. Vision 53 (2003) 225-243.

[8] C. Li, C. Xu, C. Gui, M.D. Fox, Distance regularized level set evolution and its application to image segmentation, IEEE Trans. Image Process. 19 (2010) 3243-3254.

[9] C. Xu, J.L. Prince, Snakes, shapes, and gradient vector flow, IEEE Trans. Image Process. 7 (1998) 359-369.

[10] T. Chan, L. Vese, Active contours without edges, IEEE Trans. Image Process. 10 (2001) 266-277.

[11] L. Vese, T. Chan, A multiphase level set framework for image segmentation using the Mumford-Shah model, Int. J. Comput. Vision 50 (2002) 271-293.

[12] A. Tsai, A. Yezzi, A.S. Willsky, Curve evolution implementation of the Mumford-Shah functional for image segmentation denoising, interpolation, and magnification, IEEE Trans. Image Process. 10 (2001) 1169-1186.

[13] C. Li, C. Kao J. Gore, Z. Ding, Minimization of region-scalable fitting energy for image segmentation, IEEE Trans. Image Process. 17 (2008) 1940-1949.

[14] C. Li, C. Kao, J. Gore, Z. Ding, Implicit active contours driven by local binary fitting energy, in: Proceedings of the IEEE Conference Computer Vision and Pattern Recognition, Washington, DC, USA, 2007, pp. 1-7.

[15] K. Zhang, H. Song, L. Zhang, Active contours driven by local image fitting energy, Pattern Recognit. 43 (2010) 1199-1206.

[16] S. Liu, Y. Peng, A local region-based Chan-Vese model for image segmentation, Pattern Recognit. 45 (2012) 2769-2779.

[17] L. Wang, L. He, A. Mishra, C. Li, Active contours driven by local Gaussian distribution fitting energy, Signal Process. 89 (2009) 2435-2447.

[18] W. Liu, Y. Shang, X. Yang, Active contour model driven by local histogram fitting energy, Pattern Recognit. Lett. 34 (2013) 655-662.

[19] Z. Ji, Y. Xia, Q. Sun, G. Cao, Q. Chen, Active contours driven by local likelihood image fitting energy for image segmentation, Inf. Sci. 301 (2015) 285-304.

[20] Q Ge, L. Xiao, J. Zhang, Z. Wei, A robust patch-statistical active contour model for image segmentation, Pattern Recognit. Lett. 33 (2012) 1549-1557.

[21] Q. Wu, J. Fang, Parametric kernel-driven active contours for image segmentation, J. Electron. Imaging. 21 (2012) 043015.

[22] K. Ding, L. Xiao, G. Weng, Active contours driven by region-scalable fitting and optimized Laplacian of Gaussian energy for image segmentation, Signal Process. 134 (2017) 224-233.

[23] L. Wang, C. Li, Q. Sun, D. Xia, C. Kao, Active contours driven by local and global intensity fitting energy with application to brain MR image segmentation, Comput. Med. Imaging Graphics 33 (2009) 520-531.

[24] C. He, Y. Wang, Q. Chen, Active contours driven by weighted region-scalable fitting energy based on local entropy, Signal Process. 92 (2012) 587-600.

[25] Ding, Keyan, Linfang Xiao, and Guirong Weng. "Active contours driven by local prefitting energy for fast image segmentation." Pattern Recognition Letters 104 (2018): 29-36. DOI: 10.1016/j.patrec.2018.01.019

[26] Liu, Huaxiang, Jiangxiong Fang, Zijian Zhang, and Yongcheng Lin. "A Novel Active Contour Model Guided by Global and Local Signed Energy-Based Pressure Force." IEEE Access 8 (2020): 59412-59426. DOI: 10.1109/ACCESS.2020.2981596

[27] A Dongdong Ma, Qingmin Liao. ZiqinChen, Ran Liao, Hui Ma, “Adaptive local-fittingbased active contour model for medical image segmentation”, Signal Processing: Image Communication 76(2019) 201-213 